\title{
APPROXIMATIONS OF THE IDENTITY OPERATOR BY SEMIGROUPS OF LINEAR OPERATORS
}

\author{
A. PAZY
}

Abstract. Let $T(t), t \geqq 0$, be a strongly continuous semigroup of linear operators on a Banach space $X$. It is proved that if for every $C>0$ there exists a $\delta_{c}>0$ such that $\|I-T(t)\| \leqq 2-C t \log (1 / t)$ for $0<t<\delta_{c}$ then $A T(t)$ is bounded for every $t>0$. It is shown by means of an example that $\|I-T(t)\| \leqq 2-C t$ for a fixed $C$ and all $0<t<\delta$ is not sufficient to assure the boundedness of $A T(t)$ for any $t \geqq 0$.

1. Introduction and statement of the results. Let $X$ be a Banach space, $T(t), 0 \leqq t<\infty$, a strongly continuous semigroup of bounded linear operators on $X$ (see Hille-Phillips [1, p. 321]). It is well known that the degree of approximation of the identity by the semigroup for small values of the parameter $t$, that is, the order of magnitude of $\|I-T(t)\|$ as a function of $t$, is closely related to regularity properties of the semigroup $T(t)$. Some early results concerning such approximations are given in $[1, \$ 10.7]$. Recently $\mathrm{J}$. Neuberger [2] proved the following result: If

$$
\limsup _{t \rightarrow 0}\|I-T(t)\|<2
$$

then $A T(t)$ is a bounded linear operator for every $t>0$, where $A$ is the infinitesimal generator of $T(t)$. T. Kato [3] generalized this result, showing that (1) implies that $T(t)$ is a holomorphic semigroup, that is, a semigroup belonging to the class $H\left(\Phi_{1}, \Phi_{2}\right)$ for some $\Phi_{1}<0<\Phi_{2}$ $[1$, p. 325] and a fortiori $A T(t)$ is bounded for every $t>0$. However, for $A T(t)$ to be bounded for every $t>0$, it is not necessary that $T(t)$ will be holomorphic. Our main result extends the result of Neuberger, showing that even if $\lim \sup _{t \rightarrow 0}\|I-T(t)\|=2$ but $\|I-T(t)\|$ does not approach 2 too rapidly, $A T(t)$ is bounded for every $t>0$. Precisely our result is given by

THEOREM. Let $T(t)$ be a strongly continuous semigroup of bounded linear operators. If for every $C>0$ there exists a $\delta_{c}>0$ such that

Received by the editors December 28, 1970.

AMS 1969 subject classifications. Primary 4750.

Key words and phrases. Holomorphic, strongly continuous semigroups of bounded linear transformations.

Copyright (c) 1971, American Mathematical Society 


$$
\|I-T(t)\| \leqq 2-C t \log (1 / t) \quad \text { for } 0<t<\delta_{c},
$$

then $A T(t)$ is a bounded operator for every $t>0$.

By means of Lemma 1 we can also extend the corollary to Theorem A of [2] as follows.

Corollary. If $T(t)$ satisfies (2) for some fixed $C$ and $\delta_{c}$ and $T(t)$ can be extended to a group of bounded linear operators, then $A$ is bounded.

2. Proofs. Our theorem as well as the corollary is a consequence of the following lemma.

LEMMA. Let $T(t)$ be a strongly continuous semigroup of bounded operators on $X$ satisfying $\|T(t)\| \leqq M$ for $t \geqq 0$. If there exist constants $C$ and $\delta$ such that

$$
\|I-T(t)\| \leqq 2-C t \log (1 / t) \quad \text { for } 0<t<\delta,
$$

then $A T(t)$ is a bounded operator for every $t>M / C$.

Proof. Let $\alpha$ be real and $x \in D(A)$ (the domain of $A$ ). From the identity

$$
T(t) x-e^{i t \alpha} x=\int_{0}^{t} e^{i \alpha(t-s)} T(s)(A-i \alpha) x d s
$$

it follows that $\left\|\left(T(t)-e^{i t \alpha}\right) x\right\| \leqq t M\|(A-i \alpha) x\|$. Substituting $\alpha= \pm \pi / t$ we obtain $\|(I+T(t)) x\| \leqq t M\|(A \pm i(\pi / t)) x\|$. From (3) we have

$$
\|I+T(t)\| \geqq 2-\|I-T(t)\| \geqq C t \log (1 / t) \quad \text { for } 0<t<\delta_{c}
$$

and therefore

$$
\|(A-i \tau) x\| \geqq((C / M) \log (|\tau| / \pi))\|x\|
$$

where $\tau= \pm \pi / t$.

From (4) it follows that $A-i \tau$ is one-to-one for $|\tau|$ sufficiently large. We shall prove that it is on to $X$. Since $A$ is the infinitesimal generator of a uniformly bounded semigroup $(A-(\rho+i \tau))^{-1}$ exists and has domain $X$ for every $\rho>0$ and $\left\|(A-(\rho+i \tau))^{-1}\right\| \leqq M / \rho$ (see $\left[4\right.$, p. 624]). Let $f \in X$ and denote by $x_{\rho}$ the solution of $(A-(\rho+i \tau)) x_{\rho}$ $=f$. Then $\left\|x_{\rho}\right\| \leqq(M / \rho)\|f\|$ and therefore

$$
\left\|(A-i \tau) x_{\rho}\right\| \leqq \rho\left\|x_{\rho}\right\|+\|f\| \leqq(M+1)\|f\| .
$$

From (4) it then follows that $\left\|x_{\rho}\right\|$ is bounded as $\rho \rightarrow 0$ and

$$
\left\|(A-i \tau) x_{\rho}-f\right\|=\rho\left\|x_{\rho}\right\| \rightarrow 0 \quad \text { as } \rho \rightarrow 0
$$

i.e., $(A-i \tau) x_{\rho} \rightarrow f$. Using (4) again we obtain that $x_{\rho}$ is convergent to 
some element $x \in X$ as $\rho \rightarrow 0$. Since $A$ is closed it follows that $(A-i \tau) x$ $=f$. Thus $A-i \tau$ is onto, $(A-i \tau)^{-1}$ exists and

$$
\left\|(A-i \tau)^{-1}\right\| \leqq(M / C)(\log (|\tau| / \pi))^{-1} .
$$

Therefore,

$$
\limsup _{|\tau| \rightarrow \infty}(\log |\tau|)\left\|(A-i \tau)^{-1}\right\| \leqq M / C
$$

which implies by Theorem 2.2 of $[5]^{1}$ that $A T(t)$ is bounded for $t>M / C$.

Proof of the Theorem. Let $T(t)$ be a semigroup of bounded linear operators then there exist constants $M$ and $\omega \geqq 0$ such that $\|T(t)\| \leqq M e^{\omega t}\left[4\right.$, p. 619]. Consider the semigroup $S(t)=e^{-\omega t} T(t)$ then

$$
\begin{aligned}
\|S(t)-I\| & \leqq e^{-\omega t}\|T(t)-I\|+\left(e^{-\omega t}-1\right) \\
& \leqq 2-C t \log (1 / t)+\left(e^{-\omega t}-1\right) \leqq 2-C_{1} t \log (1 / t)
\end{aligned}
$$

for every $C_{1}, 0<C_{1}<C$ and $0<t<\delta\left(C_{1}\right)$. Thus if $T(t)$ satisfies the conditions of the Theorem (respectively of the Lemma) so does $S(t)$. But $S(t)$ is uniformly bounded and therefore differentiable for every $t>0$ (respectively $t>M / C_{1}$ ). This implies that $T(t)$ is differentiable for every $t>0$ (respectively $t>M / C_{1}$ ) which is equivalent to the boundedness of $A T(t)$ for every $t>0$ (respectively $t>M / C_{1}$ ).

Proof of THE Corollary. By the previous argument $A T(t)$ is bounded for $t$ large enough and therefore also $A=T(-t) A T(t)$ is bounded as the product of two bounded operators.

It is clear that under the conditions of our theorem we may have $\lim \sup _{t \rightarrow 0}\|I-T(t)\|=2$. In such a case, if $\|T(t)\| \leqq 1$ and $X$ is uniformly convex, $T(t)$ is not holomorphic. This follows from the results of Kato [3]. Nevertheless, our theorem assures that $A T(t)$ is bounded for every $t>0$.

We conclude with an example in which $\|I-T(t)\| \leqq 2-C t$ for some constant $C$ and for $0 \leqq t \leqq 1$ but $A T(t)$ is not bounded for any $t>0$.

Let $X=l_{2}$, that is, sequences $\left\{\alpha_{n}\right\}_{n=1}^{\infty}$ of complex numbers with the norm $\left\|\left\{\alpha_{n}\right\}\right\|=\left(\sum_{n=1}^{\infty}\left|\alpha_{n}\right|^{2}\right)^{1 / 2}$. Let

$$
T(t)\left\{\alpha_{n}\right\}=\left\{\beta_{n}(t) \alpha_{n}\right\}
$$

where $\beta_{n}(t)=\exp \left[\left(-\log (n+1)+i n^{n}\right) t\right]$. Clearly $T(t)$ is a strongly continuous semigroup on $X$ and

$\|I-T(t)\|=\sup _{n \geqq 1}\left|1-\beta_{n}(t)\right| \leqq 1+e^{-t \log 2} \leqq 2-C_{0} t, \quad 0 \leqq t \leqq 1$,

1 The theorem in [5] is stated with $\mu>\omega$ but it is clear from the proof that if $R\left(\mu+i_{\tau}: A\right)$ exists for $\mu=\omega$ the result is the same. In our case $\mu=\omega=0$. 
where $C_{0}=\frac{1}{2} \log 2$. On the other hand,

$$
\|A T(t)\|=\sup _{n \geqq 1}\left|-\log (n+1)+i n^{n}\right| e^{-t \log n} \geqq \sup _{n \geqq 1} n^{n-t}=\infty .
$$

Thus, for no $t \geqq 0, A T(t)$ is bounded.

\section{REFERENCES}

1. E. Hille and R. S. Phillips, Functional analysis and semi-groups, rev. ed., Amer. Math. Soc. Colloq. Publ., vol. 31, Amer. Math. Soc., Providence, R. I., 1957. MR 19, 664.

2. J. W. Neuberger, Analyticity and quasi-analyticity for one parameter semigroups, Proc. Amer. Math. Soc. 25 (1970), 488-494.

3. T. Kato, A characterization of holomorphic semigroups, Proc. Amer. Math. Soc. 25 (1970), 495-498.

4. N. Dunford and J. T. Schwartz, Linear operators. I: General theory, Pure and Appl. Math., vol. 7, Interscience, New York, 1958. MR 22 \#8302.

5. A. Pazy, On the differentiability and compactness of semi-groups of linear operators, J. Math. Mech. 17 (1968), 1131-1141. MR 37 \#6797.

Hebrew University, JERUSALEM, ISRAEL 